\title{
A Method for Motion Detection and Categorization in Perfusion Weighted MRI
}

\author{
Rohit Gautam \\ CVIT, IIIT-Hyderabad \\ Hyderabad, India \\ rohit.gautam@research.iiit.ac.in
}

\author{
Jayanthi Sivaswamy \\ CVIT, IIIT-Hyderabad \\ Hyderabad, India \\ jsivaswamy@iiit.ac.in
}

\author{
Ravi Varma \\ KIMS Hospital \\ Hyderabad, India \\ varmaji@rediffmail.com
}

\begin{abstract}
The blood perfusion measurement is done by injecting a bolus of contrast agent in the brain followed by imaging over a period of time (scan). This process can extend into minutes and hence any patient motion mid-scan results in corrupted data. This is often observed in dynamic magnetic resonance (MR) imaging for both susceptibility (DSC) and contrast enhanced (DCE) scans. Motion correction done after scanning is typically the most time-intensive step in the entire measurement process since it involves registering each volume in the time-series to a reference volume. We argue that detecting the presence of motion prior to correction can mitigate this problem by reducing the number of volumes to be corrected. The challenge in motion detection is that the injected contrast alters the signal intensity as a function of time leading to false alarms. We present a robust multi-stage method: subdivision of the time series data into bolus and non-bolus phases; clustering-based identification of bolusaffected pixels followed by correction of their intensity using a Gamma variate function fitting-based method and a 2D block-wise phase correlation for detecting motion between adjacent volumes in DSC-MRI data. The proposed method was tested on a DSC MR sequence with simulated motion of varying degrees. The experimental results show that the entropy of the derived motion fields is a good metric for detecting and categorizing the motion. The proposed scheme when applied prior to correction can achieve on average a $37 \%$ reduction in the time required for motion correction.
\end{abstract}

\section{Keywords}

motion detection, 4D imaging, dynamic susceptibility contrast (DSC) MRI, dynamic contrast enhanced (DCE) MRI, phase correlation, gamma-variate-function, intensity correction.

Stroke is a rapid loss in brain function due to disturbance in blood supply to the brain. Usually there is a delay from

${ }^{*}$ Corresponding author

Permission to make digital or hard copies of all or part of this work for personal or classroom use is granted without fee provided that copies are not made or distributed for profit or commercial advantage and that copies bear this notice and the full citation on the first page. To copy otherwise, to republish, to post on servers or to redistribute to lists, requires prior specific permission and/or a fee.

ICVGIP'12, December 16-19, 2012, Mumbai, India

Copyright 2012 ACM 978-1-4503-1660-6/12/12 ...\$15.00. the onset of symptoms to permanent tissue damage. During this window, a part of the tissue at risk can be salvaged. Hence, an early diagnosis can help reduce mortality and morbidity of patients. This idea has led to advancements of new and effective methods for early diagnosis and treatment of stroke. Magnetic resonance imaging(MRI) is increasingly being used for diagnosis of acute ischaemic stroke. Improvements in MRI has led to perfusion MRI for treatment of acute ischaemic stroke. Cerebral perfusion is commonly studied using T2*-weighted dynamic susceptibility (DSC) imaging or T1-weighted dynamic contrast enhancement (DCE) imaging. An exogenous paramagnetic contrast agent is injected into the blood stream of patient and it is tracked in the brain (hence the term 'bolus tracking') by acquiring 4D time series MRI dataset [6][13]. Perfusion measurements with MRI is also used in other areas like tumour characterization and progression, determining salvageable tissues post acute ischaemic event in brain, inflammation and infectious diseases.

Once the time-varying data is obtained, it is parametrized on voxel-by-voxel basis using various physiological models to obtain various hemodynamic indices, like cerebral blood flow(CBF), cerebral blood volume(CBV), mean transit time (MTT). These indices help profile the blood flow characteristics in different tissues which is needed in detection of various disease states and deciding treatment options [6][4][17]. In perfusion weighted imaging (PWI), a series of volumes are acquired resulting in an extension in the data acquisition time. Patient motion is often observed during this timeperiod. Accurate measurement of perfusion parameters relies on tracking the signal in a particular voxel which is compromised due to patient motion. This motion causes intervolume misalignments and mixing of signals from neighbourhood tissues. Consequently, the perfusion parameters generated from this data become inaccurate. Figure 1 and Table 1 demonstrate the effect of patient motion on perfusion parameters - time-to-peak (TTP) and cerebral blood volume (CBV) depending on the degree of motion. These were estimated using the perfusion mismatch analyser(PMA) tool developed by ASIST-Japan [9]. From these plots, it is seen that with an increase in the degree of motion, the parameter estimation error increases with the error percentage exceeding $50 \%$ for TTP while it remains under $40 \%$ for CBV. Motion correction is typically done after the data has been acquired since the image acquisition time is large and the contrast agent has to be used again. Thus an accurate, reliable motion detection and correction method becomes necessary in such cases. The underlying challenge for these 


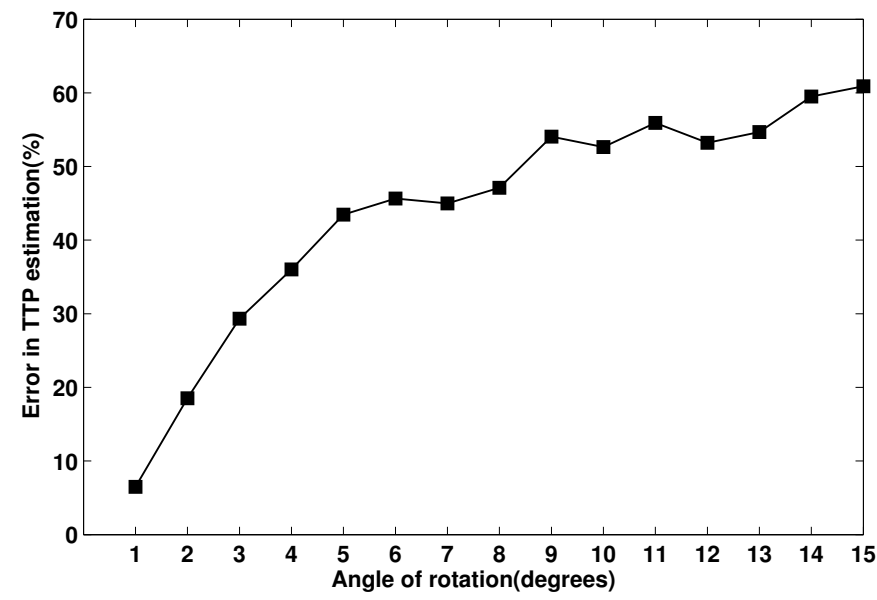

(a) TTP Plot

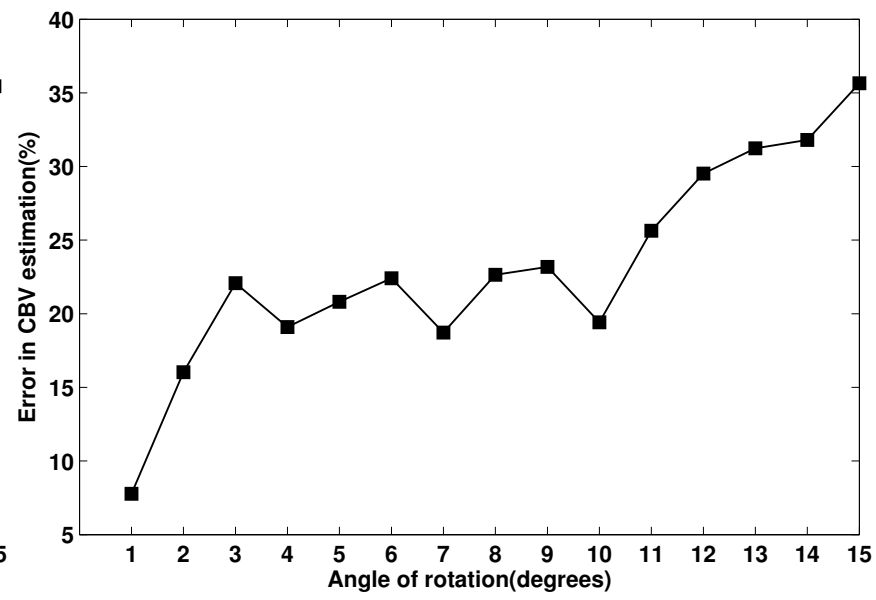

(b) CBV Plot

Figure 1: Variation in estimation of parameters - TTP and CBV with different degrees of rotation.

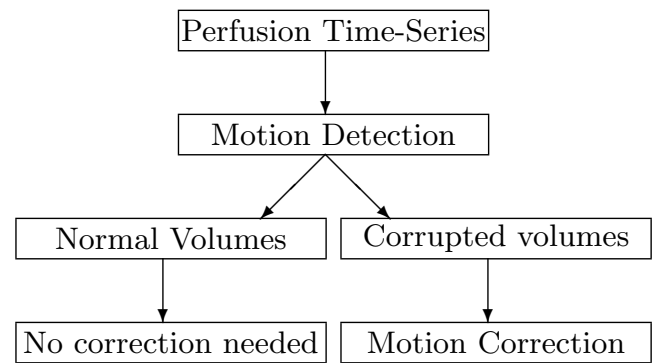

Figure 2: Proposed motion correction framework for perfusion weighted MRI.

algorithms with perfusion MRI is the localised variation in the signal in the post-wash-in stages of the bolus in the timeseries volumes. This variation causes traditional methods of motion detection to perform poorly.

The standard approach to motion correction of $4 \mathrm{D}$ perfusion MRI data perform is via an alignment of motion corrupted volumes. Thus, there is no explicit detection and subsequent correction of motion. For DSC-MRI, motion correction techniques include registering the time series to either a single volume or the mean volume of the entire time-series data[7]. [2] includes a model of dynamic contrast in an iterative registration process for tracking tumour motion. 3D rigid registration via cylindrical phase correlation has been proposed in [1] which is capable of handling highly misaligned volumes and is noise resilient. In DCEMRI, motion correction has been done via registration techniques including a rigid body model [10], nonrigid B-spline [20], maximization of a special Gibbs energy function using a gradient descent algorithm [5], mutual information with a spatial transformation model [14], an intensity correction model [11], group-wise registration [8] or progressive principal component registration [12]. In general, motion correction has been found to be a time-limiting step ( $\sim 90 \%$ of processing time) in a PWI analysis pipeline [16]. Since $3 \mathrm{D}$ registration is computationally intensive [15] it would be more efficient if only a subset of volumes need to be aligned instead of every volume in the entire time-series data. In
Table 1: Clinical parameters - Time to peak (TTP) and CBV for a ROI for 16 datasets with different degrees of motion ( $A_{\theta}$ denotes parameter $A$ estimated at angle of rotation $\theta^{\circ}$.)

\begin{tabular}{|c|c|c|c|c|}
\hline $\begin{array}{c}\text { Angle of } \\
\text { rotation } \\
\text { (in degrees) } \\
\end{array}$ & $\begin{array}{l}\text { TTP } \\
(\text { sec) }\end{array}$ & $\begin{array}{c}\text { Difference } \\
\text { from normal } \\
\left(\mathbf{T T P}_{\theta}-\mathrm{TTP}_{0}\right)\end{array}$ & $\begin{array}{l}\text { CBV } \\
\left(\mathrm{cm}^{3}\right)\end{array}$ & $\begin{array}{c}\text { Difference } \\
\text { from normal } \\
\left(\mathrm{CBV}_{\theta}-\mathrm{CBV}_{0}\right)\end{array}$ \\
\hline 0 & 24.07 & \begin{tabular}{|l|}
0 \\
\end{tabular} & 24.46 & \begin{tabular}{|c|}
0 \\
\end{tabular} \\
\hline 1 & 25.63 & 1.56 & 22.56 & 1.90 \\
\hline 2 & 28.53 & 4.46 & 20.54 & 3.92 \\
\hline 3 & 31.13 & 7.06 & 19.06 & 5.40 \\
\hline 4 & 32.74 & 8.67 & 19.79 & 4.67 \\
\hline 5 & 34.53 & 10.46 & 19.37 & 5.09 \\
\hline 6 & 35.06 & 10.99 & 18.98 & 5.48 \\
\hline 7 & 34.90 & 10.83 & 19.88 & 4.58 \\
\hline 8 & 35.41 & 11.34 & 18.92 & 5.54 \\
\hline 9 & 37.08 & 13.01 & 18.79 & 5.67 \\
\hline 10 & 36.74 & 12.67 & 19.71 & 4.75 \\
\hline 11 & 37.53 & 13.46 & 18.19 & 6.27 \\
\hline 12 & 36.88 & 12.81 & 17.24 & 7.22 \\
\hline 13 & 37.23 & 13.16 & 16.82 & 7.64 \\
\hline 14 & 38.39 & 14.32 & 16.68 & 7.78 \\
\hline 15 & 38.73 & 14.66 & 15.74 & 8.72 \\
\hline
\end{tabular}

general, if a time-series has $\mathrm{N}$ phases (or volumes), all the phases are not corrupted by motion. Hence, it is worthwhile to first detect the subset $\leq \mathrm{N}$ volumes that is affected by motion and subsequently correct this subset. An example of such a motion correction framework is shown in Figure 2. In this paper, we present a framework for fast motion detection in DSC-MRI

\section{METHOD}

In perfusion studies, the injected contrast agent takes some time to reach the region of interest (wash-in), pass through and eventually pass out (wash-out) of the region. Hence, the given $4 \mathrm{D}$ time-series data can be divided into 3 distinct sets depending on the status of bolus in the ROI: volumes belonging to 1) pre-wash-in, 2) between-wash-in-and-wash-out and 3) post wash-out sets. In the next section, we describe gamma-variate-function (GVF) fitting on perfusion curve to 


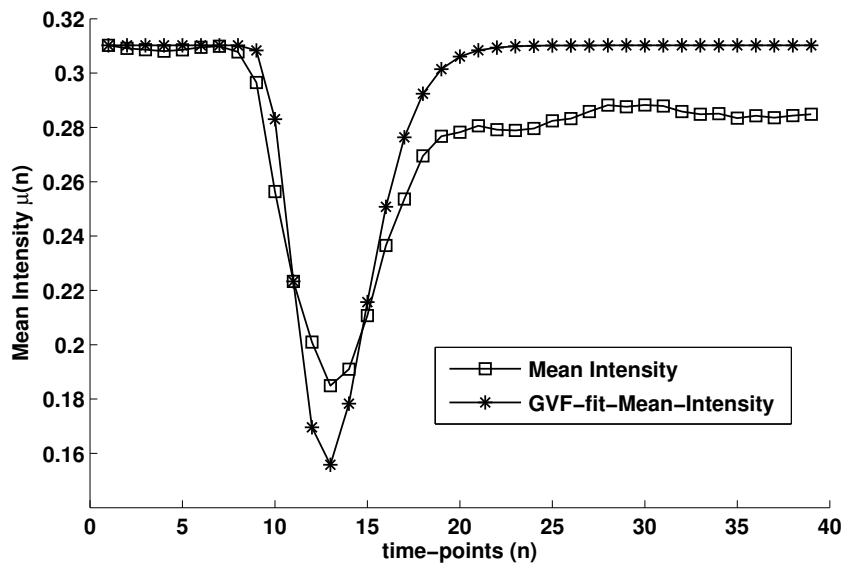

Figure 3: Gamma-variate-function (GVF) fitting on the mean-intensity curve of a DSC-MRI time-series.

divide the perfusion time-series into above mentioned sets.

\subsection{Gamma-variate-function(GVF) fitting}

The given PWI time series of volumes $\left\{F_{n} ; n=1 . . N\right\}$ is first divided into 3 distinct sets based on the status of the bolus in the brain. In order to identify these sets we use the gamma-variate function [3] which models the transverse relaxation rate change-time curves, $\Delta R_{2}(t)$ or $\Delta R_{2}^{*}(t)$, with the first-pass(at wash-in time) of the bolus. This function is given as:

$$
\Delta R_{2}^{*}(t)=A\left(t-t_{0}\right)^{\alpha} e^{-\left(t-t_{0}\right) / \beta}, t>t_{0}
$$

where, $\Delta R_{2}^{*}(t)$ is the transverse relaxation rate of the magnetic field, $t_{0}$ is the wash-in time point of the bolus, and $\mathrm{A}$, $\alpha$ and $\beta$ are parameters that decide the shape of function. Due to the bolus, the signal intensity $\mathrm{s}(\mathrm{t})$ changes in an exponential manner depending on the transverse and longitudinal relaxation rate changes, $\Delta R_{2}^{*}$ and $\Delta R_{1}$ respectively, [13] as:

$$
s(t)=s\left(t_{0}\right)\left(1-e^{T R \cdot \Delta R_{1}(t)}\right) \cdot e^{T E \cdot \Delta R_{2}^{*}(t)}
$$

where, $s\left(t_{0}\right)$ is the baseline signal intensity, TR is the repetition time and TE is the echo time. Hence, an accurate fitting of $\Delta R_{2}^{*}$ curve gives an accurate change in signal intensity $\mathrm{s}(\mathrm{t})$.

The proposed GVF fitting begins with the extraction of the mean intensity of each volume. This results in a curve $\mu_{a}(n)$. The gamma-variate function (GVF) in Eqn.1 is next fit to $\mu_{a}(n)$ which results in GVF-fit-mean-intensity curve $\left(\mu_{g}(n)\right)$. The wash-in $\left(n_{w i n}\right)$ and wash-out $\left(n_{\text {wout }}\right)$ time points are then determined using this curve as points of steep negative and positive transitions. It should be noted that estimating these points without the GVF fitting, while possible, will yield only rough estimates. These points help divide the given $4 \mathrm{D}$ dataset into three sets: Set 1 of prewash-in, Set 2 of transit and Set 3 of post-washout stage of the bolus passage. Figure 3 shows an example of GVFfitting on the mean-intensity curve of a perfusion series of 40 time-points. GVF-fitting here models the normal behaviour of contrast agent when passing through vasculature. In the following section, we describe the traditional phase correlation technique.

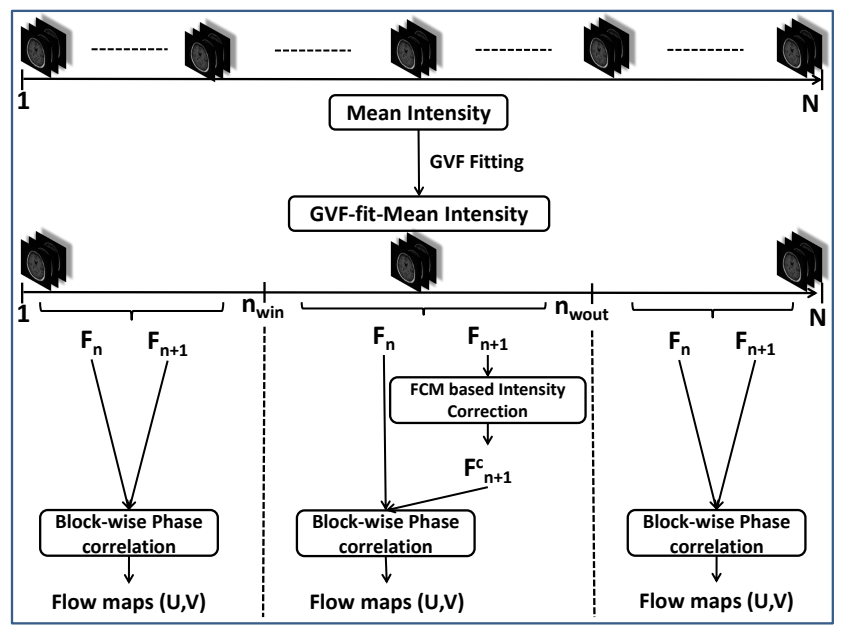

Figure 4: Motion Detection Scheme for a DSC-MRI time-series.

\subsection{Phase Correlation}

Phase correlation [1] is a technique that has been used for $3 \mathrm{D}$ registration. It is based on the Fourier shift theorem which states that a shift in the spatial domain of a signal corresponds to a linear phase shift in the Fourier domain.

Let $g_{a}(x): \mathbb{R} \rightarrow \mathbb{R}$ and $g_{b}(x): \mathbb{R} \rightarrow \mathbb{R}$ be two functions that are related as follows:

$$
g_{b}(x)=g_{a}\left(x-x_{0}\right)
$$

Then, according to Fourier shift theorem,

$$
\mathfrak{F}\left\{g_{a}\left(x-x_{0}\right)\right\}=G_{a}(\omega) e^{-j\left(\omega x_{0}\right)}
$$

where $G(\omega)$ denotes $\mathfrak{F}\{g(x)\}$, the Fourier transform of $\mathrm{g}(\mathrm{x})$. Hence,

$$
G_{b}(\omega)=G_{a}(\omega) e^{-j\left(\omega x_{0}\right)}
$$

The normalized cross-power spectrum $\left(S_{a b}(\omega)\right)$ is

$$
S_{a b}(\omega)=\frac{G_{a} G_{b}^{*}}{\left|G_{a}\right|\left|G_{b}^{*}\right|}=e^{j\left(\omega x_{0}\right)}
$$

The inverse Fourier transform of this function is an impulse which gives the flow offset.

$$
\begin{aligned}
r(x, y) & =\mathfrak{F}^{-1}\left\{S_{a b}(\omega)\right\} \\
& =\delta\left(x-x_{0}\right)
\end{aligned}
$$

Phase correlation thus enables determining the degree of translation between two images. A rotation between a pair of images can be visualized as a translation of pixels from location $(\mathrm{x}, \mathrm{y})$ in the first image to a new location $(\mathrm{x}-\Delta \mathrm{x}$, $\mathrm{y}-\Delta \mathrm{y})$ on the second image. Hence, the above approach can be used to determine the degree of motion. In the following section, we describe our approach to determine motion between adjacent volumes in DSC-MRI time-series data.

\subsection{Proposed Motion Detection}

Given a PWI time series, we identify the volumes corrupted by motion. Since the scan time for a volume in DSC-MRI series is quite short $(\sim 2-3$ seconds $)$, it is fair to assume that there is no intra-volume motion present in the time-series. Consequently, every slice of a volume will be 


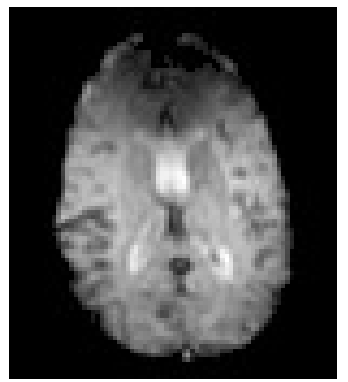

(a) $I_{n}$

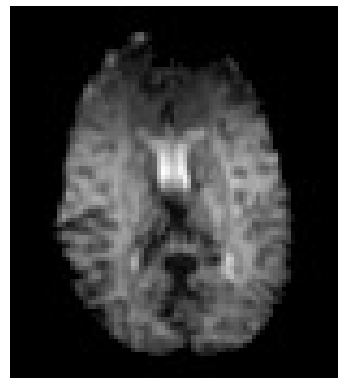

(b) $\mathbf{I}_{\mathbf{m}}$

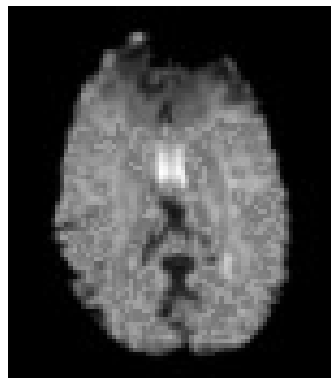

(c) $\mathbf{I}_{\mathrm{m}}^{\mathrm{c}}$

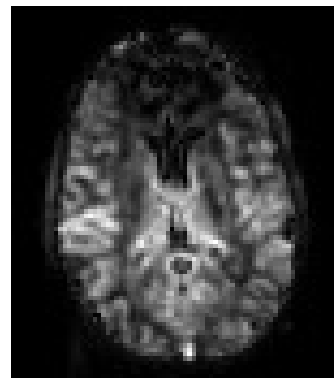

(d) $\left|\mathbf{I}_{\mathbf{n}}-\mathbf{I}_{\mathbf{m}}\right|$

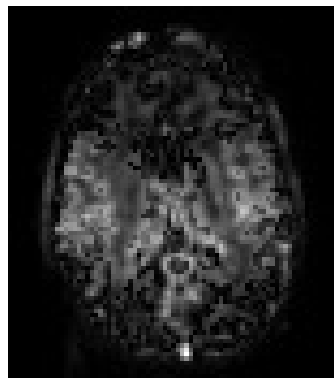

$(\mathbf{e})\left|\mathbf{I}_{\mathbf{n}}-\mathbf{I}_{\mathbf{m}}^{\mathbf{c}}\right|$

Figure 5: Example of intensity correction for original(non-motion-corrupt) DSC-MRI data. Two central slices $I_{n}$ and $I_{m}$ extracted from volumes $F_{n}$ and $F_{m}$ are shown in (a) and (b) respectively. (c) is the intensity corrected image of (b). Notice that there is a reduction in absolute intensity difference in (e) as compared to (d). Ideally, the absolute difference in (e) should be zero.

corrupted by the same degree of motion. Hence, we detect motion in the time-series using the central slices in every volume. Figure 4 shows the block diagram for the proposed motion detection technique. In the first stage, the time-series is divided into 3 sets based on the status of bolus in brain, using GVF fitting. Next, motion is detected in each of these sets. Our motion detection method is based on intensity correction and block-wise phase correlation. The method for sets 1 and 3 (which correspond to pre-wash-in and postwash-out) differs slightly from that of set 2 (between-washin-and-wash-out) due to the presence of bolus in set 2 . In the next section, we explain the proposed motion detection strategy for sets 1 and 3.

\subsubsection{Motion Detection for Sets 1 and 3}

As the bolus is not present in sets 1 and 3 , intensity correction is not required in these sets and motion can be detected in the volumes of these sets using phase correlation. Given a pair of adjacent volumes $\left(F_{n}, F_{n+1}\right)$ in a set, we consider only their central slices $\left(I_{n}, I_{n+1}\right)$ and compute the motion field $\left(U_{n}, V_{n}\right)$ between them. In the absence of any motion between the volumes, this field will be uniformly zero. The motion field is computed by considering a block of pixels $b(i, j)$ around every pixel at location $(i, j)$ in the slice pair and performing a phase correlation. To handle the inter-slice intensity variation that is characteristic of DSC-MRI, the blocks are normalised first by shifting the mean pixel value of every block to zero. These normalised blocks are $\tilde{b}_{n}=b_{n}-\bar{b}_{n}$ where $\bar{b}$ denotes the mean pixel value in the block. Phase correlation is applied to the pair $\left(\tilde{b}_{n}(i, j), \tilde{b}_{n+1}(i, j)\right)$ and the desired flow vector $(u(i, j), v(i, j))$ is found from the locations of maxima of the cross power spectrum $S_{\tilde{b}_{n}} \tilde{b}_{n+1}$ The flow maps $U_{n}=u(i, j)$ and $V_{n}=v(i, j)$ describing the motion between $I_{n}$ and $I_{n+1}$ are given by:

$$
(u, v)=\operatorname{argmax}_{(i, j) \varepsilon b_{i, j}}\left(\mathfrak{F}^{-1}\left\{S_{\tilde{b}_{n}} \tilde{b}_{n+1}\right\}\right)
$$

where, $S_{\tilde{b}_{n} \tilde{b}_{n+1}}=\frac{\tilde{B}_{n} \tilde{B}_{n+1}^{*}}{\left|\tilde{B}_{n}\right|\left|\tilde{B}_{n+1}^{*}\right|}$ and $\tilde{B}=\mathfrak{F}\{\tilde{b}\}$. Next, we present the proposed motion detection strategy for set 2 .

\subsubsection{Motion Detection for Set 2}

Since the bolus is present in volumes in set 2, intensity correction is required prior to motion detection here. In
DSC-MRI, as the contrast agent passes through some regions in the brain, it results in signal attenuation only in those regions. This leads to a non-uniform intensity change within the volume. We identify these bolus-affected regions using a fuzzy c-means (FCM) clustering method. This technique aids in segmenting a DSC-MRI volume $F$ into normal $\left(F_{\text {normal }}\right)$ and bolus affected $\left(F_{\text {bolus }}\right)$ regions. To account for changes in image intensity across time points, we use the above GVF fitting on the mean-intensity perfusion curve $\left(\mu_{g}(n)\right)$, to obtain the intensity change across time points. The intensity correction is then applied to only bolus affected regions. The intensity corrected volume is generated as follows. For convenience, the volume $F$ at time point $n$ is now denoted as $F(n)$ :

$$
\begin{aligned}
F_{\text {bolus }}^{c}(n+1) & =F_{\text {bolus }}(n+1) \frac{\mu_{g}(n)}{\mu_{g}(n+1)} \\
F^{c}(n+1) & =F_{\text {normal }}(n+1) \cup F_{\text {bolus }}^{c}(n+1)
\end{aligned}
$$

where the superscript $c$ denotes the corrected result. Figure 5 shows an example of intensity correction on a pair of slices with no motion between them. As compared to Figure 5(d), Figure 5(e) shows a reduction in absolute difference in image intensity after intensity correction, which should ideally be zero. Nevertheless, the attenuation effect of bolus is minimised and this aids the correct estimation of motion between volumes.

After intensity correction, we consider the central slices $\left(I_{n}, I_{n+1}^{c}\right)$ of volumes $\left(F_{n}, F_{n+1}^{c}\right)$ and carry out motion detection in the same manner as explained for Sets 1 and 3 .

In our implementation, initially, a block size of $32 \times 32$ was used for a slice of size $128 \times 128$. In an attempt to speed up computations, various downsampling factors of the central slice with a corresponding reduction in block size were also tested. The maximum downsampling factor achieved was 4 to obtain slice of size $32 \times 32$ and the block size was reduced to $8 \times 8$.

\subsection{Motion Categorization}

The flow maps $U_{n}$ and $V_{n}$ describe the orthogonal components of the flow field relating two representative slices $\left(I_{n}\right.$ and $I_{n+1}$ ) of the bolus-phase volumes of the time-series containing $\mathrm{N}$ volumes. In the absence of any motion, these flow maps, when viewed as images, will be uniformly smooth (all pixel values are equal). Presence of motion will introduce a disturbance in this map which can be characterized in terms 
of entropy in the each of these flow maps.

To ensure that noise related changes outside the brain tissue did not affect the motion metrics, a mask for the brain region was determined for each slice using a phase-field based segmentation of the bolus-time-slice [19]. A mask was generated individually for $I_{n}$ and $I_{n+1}$. The union of the two masks was taken to ensure that entire span of motion in the two slices was captured by the mask. An entropy-based metric was used to quantify the degree of motion as follows: For a given time series $\left\{F_{n} ; n=1 . . N\right\}$, the flow fields $\left(U_{n}, V_{n}\right)$ are computed first and the peak entropy $H_{\text {peak }}$ of the flow fields is next found as

$$
\begin{aligned}
H_{n} & =H_{U_{n}}+H_{V_{n}} \\
H_{\text {peak }} & =\max \left(H_{n}\right), 1 \leq n \leq N-1
\end{aligned}
$$

where $H_{A}$ denotes the Shannon entropy of $A$. The Shannon entropy of a discrete random distribution $\mathrm{p}(\mathrm{x})$ is given by:

$$
H(p)=-\sum_{x} p(x) \log _{2} p(x)
$$

where, $\mathrm{p}(\mathrm{x})$ refers to the distribution of intensities in an image.

\section{EXPERIMENTS AND RESULTS}

\subsection{Dataset}

A DSC-MRI dataset was acquired from a 1.5T GE MRI scanner with number of volumes $=40(1 \mathrm{~s} /$ phase $)$, number of slices $=20$, slice thickness $=5 \mathrm{~mm}$, slice size $=128 \times 128$. All the computations were performed on Intel(R) Core(TM) i7-2600 CPU (3.40GHz) with 16GB RAM in MATLAB software.

In our experiments, simulated motion was applied to real perfusion data as acquiring real motion corrupted data is difficult. For validating the proposed method, a set of experiments were performed. Known amount of 3D-rotation was added to the volumes to simulate actual patient motion during DSC-MRI scan, in consultation with a neuroradiologist. A number of consecutive volumes at repeated intervals in the time-series were rotated by the same amount, which simulates a typical patient behaviour (sudden head rotation followed by remaining in that position for a period of time) inside the scanner. For the DSC-MRI dataset of $\mathrm{N}=40$ volumes, motion was added to $N_{1}=16$ volumes in $\mathrm{m}=3$ blocks of contiguous volumes: $\{[3,7],[12,16],[27,32]\}$. The rotations were kept in the range $\left[0^{\circ}, 15^{\circ}\right]$ in the transverse direction $\left(R_{z}\right)$ and in the range $\left[0^{\circ}, 10^{\circ}\right]$ in the coronal $\operatorname{direction}\left(R_{x}\right)$. For convenience in analysis, the motion corrupted volumes were categorized into different motion categories - none, minimal, medium and severe corrupted on the basis of angle of rotation. Table 2 shows different motion categories and their range of angles of rotation. This serves as ground truth for our experiments. All the results have been validated against this ground truth.

\subsection{Results}

We first present qualitative results (derived flow maps) for a pair of slices followed by quantitative results derived for the acquired dataset. Figure 6 demonstrates the phasecorrelation based flow maps for different motion categories. As seen in Figure 6(a) (a no motion case), despite changes in the signal intensities, the flow maps are almost uniform, indicating that the proposed approach is mostly immune to
Table 2: Different motion categories and their range of angles of rotation

\begin{tabular}{|c|c|}
\hline $\begin{array}{c}\text { Motion } \\
\text { Category }\end{array}$ & $\begin{array}{c}\text { Angle of Rotation } \\
\text { (degrees) }\end{array}$ \\
\hline no motion & 0 \\
\hline minimal motion & {$[1,5]$} \\
\hline mild motion & {$[6,10]$} \\
\hline severe motion & {$[11,15]$} \\
\hline
\end{tabular}

bolus-contrast based signal changes. Further, as seen in Figure $6(\mathrm{~b})$, the rotational motion creates disturbance in otherwise uniform flow maps even though there is no change in signal between two adjacent slices. The rotation of the bolus slice in Figure 6(c) (a severe motion case) creates a disturbance in the flow maps in areas which experience motion. Notice that despite changes in contrast (visible in areas around ventricles, Figure 6), the flow maps are uniform, indicating that the motion detection scheme is robust to contrast agent-related gradual signal changes.

Figure 7 demonstrates the variation of the net-entropy metric $\left(H_{n}\right)$ depending on the degree of motion between the slices for an entire time series (shown on the left) for a case with severe motion. This information can be gleaned from the corresponding net-entropy profile per slice pair (shown on the right) which varies rapidly. It can be observed that for slice pairs between which there is no motion, the net entropy is zero, while for others, the entropy reflects the degree of motion. A more detailed picture of the entropy variation across bolus-phases for 16 different datasets is presented in Table 3. Here, the first column is the ground truth information. When the ground truth motion categories are compared with the peak entropy values, it is apparent that there is a mapping between them. Hence, it is possible to classify the data into different motion categories using the peak entropy $\left(H_{\text {peak }}\right)$. As indicated in Table 3 and Figure 8 , the technique is sensitive to all categories of motion although for very small rotations (below $3^{\circ}$ ), it is difficult to distinguish it from no-motion case as the amount of entropy is mostly zero, resulting in poor sensitivity for this kind of motion. The sensitivity to detection is dependent on the image resolution and block size used for computations. In our experiments, the sensitivity to minimal motion was found to improve when the slice size was restored to the original size of $128 \times 128$. The sensitivity also increased when the block size was reduced from $32 \times 32$ to $16 \times 16$ for slice resolution $128 \times 128$. These were confirmed by visual inspection of the motion affected time-points. The increased sensitivity however, incurs an additional computational cost. Table 4 shows a detailed picture of time-taken for motion detection at different slice resolutions and block sizes. The average computation time per bolus-volume pair was $0.20 \mathrm{sec}$ for a slice of size $32 \times 32$ (0.01 sec for down-sampling, $0.19 \mathrm{sec}$ for deriving flow maps $U_{n}$ and $V_{n}$ ) which increased to $3.48 \mathrm{sec}$ for a slice of size $128 \times 128$. Thus, the overhead in computation is $\sim 17 \times$ for a $4 \times$ change in resolution and block size.

Figure 9 illustrates the effect of intensity correction on motion detection using the net-entropy $\left(H_{n}\right)$ plot for time series of 40 volumes with mild motion. To recall, the bolus phase is where the intensity correction is done. In the plot the bolus stage is between $n=9$ to 20 . Two things can be observed from this plot: With no intensity correction, the 


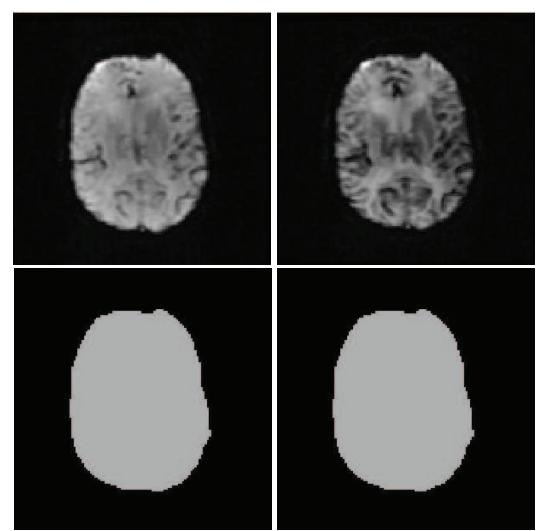

(a) No motion case

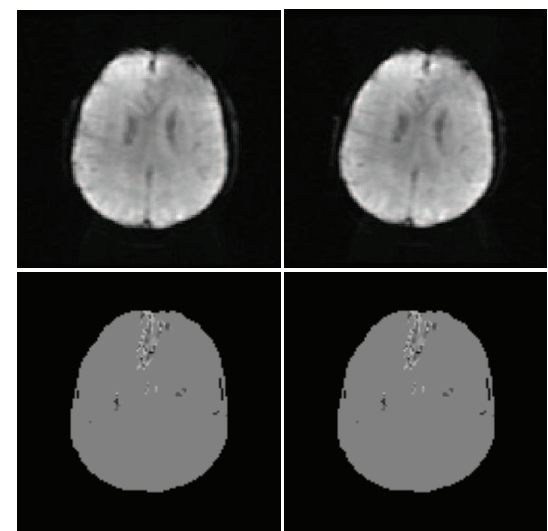

(b) Medium Motion case
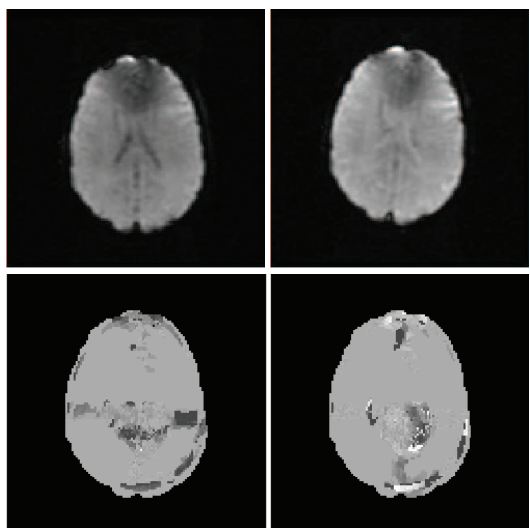

(c) Severe motion case

Figure 6: Top row shows 3 sample slice pairs for different degrees of motion. Corresponding $U_{n}$ and $V_{n}$ flow maps are shown in the bottom row.

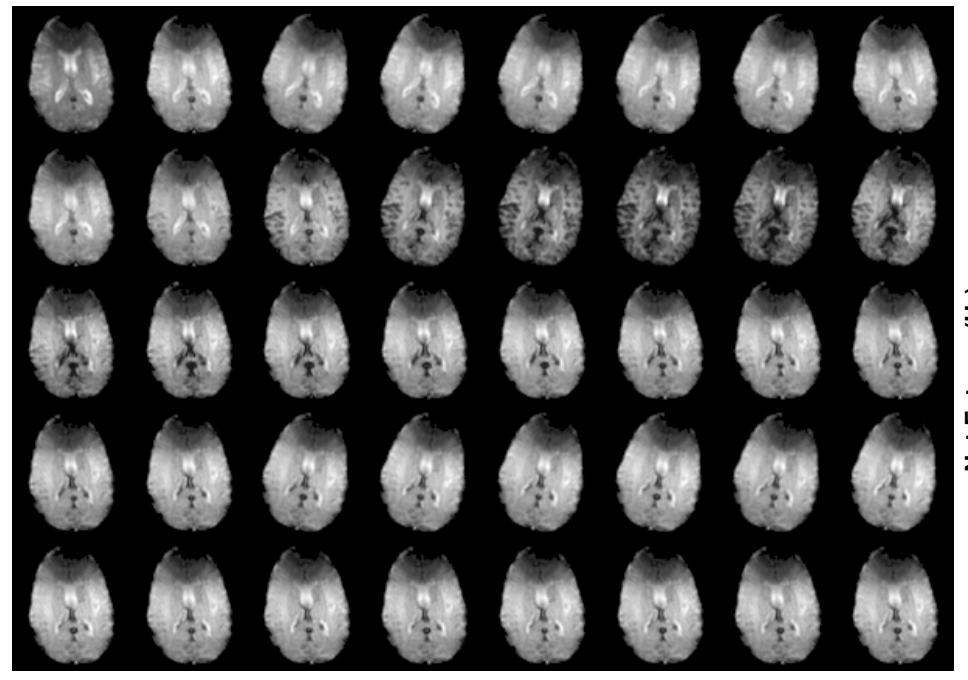

(a) DSC-MRI Time series

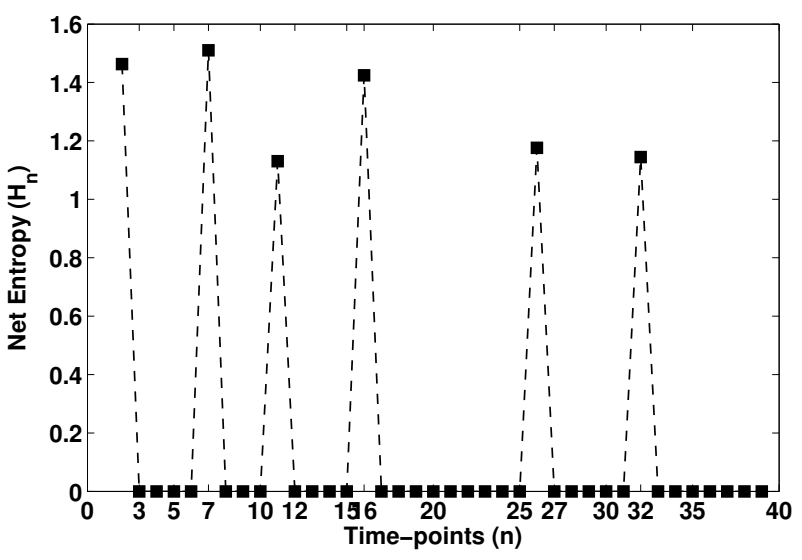

(b) Net-Entropy plot

Figure 7: Net-entropy $\left(H_{n}\right)$ profile for a severe motion case. DSC perfusion data slices are shown across bolus-phases for $n=1$ to 40 from left to right and top to bottom. First point in graph is the net entropy between slice 1 and slice 2 and so on.)

net entropy value is i) higher in some of the motion-affected volumes across the time series and ii) it is non-zero in adjacent stationary volumes only during the bolus phase. This is due to the fact traditional $2 \mathrm{D}$ block-wise phase correlation recognizes the variation in signal intensity as motion and gives a false positive result. On employing intensity correction, the net-entropy values for adjacent stationary volumes become zero as expected.

As mentioned earlier, existing techniques do not use a motion detection strategy in the correction pipeline for perfusion weighted MRI. However, we argue that motion detection prior to correction can lighten the load for the correction stage. We demonstrate this by estimating the reduction in time that can be achieved by including our detection as a front end to existing motion correction algorithms. Motion correction via $3 \mathrm{D}$ image registration methods is a standard approach followed by existing methods. This serves to align volumes in the given time-series to a chosen reference vol- ume [10][16][18]. For the purpose of our study, the volume at time-point $\mathrm{n}=2$ is chosen as the reference volume $\left(F_{R}\right)$. All the remaining volumes in the time-series are registered to this reference volume. Table 5 shows the time taken by some existing methods of motion correction algorithms for perfusion weighted MRI. In our DSC-MRI time-series data of $N=40$ volumes, motion was added to $N_{1}=16$ volumes. However, this was done only to $m=3$ blocks of contiguous volumes. Initially, all the remaining $N-1=39$ volumes are registered to reference volume $F_{R}$. With motion detection, the number of volumes that need to be registered reduces to $23\left((N-1)-N_{1}+m\right)$. The reduction occurs since the volumes in each of these $m=3$ intervals undergo same degree of motion, only one volume in each interval needs to be aligned. This results in a reduction in time taken for motion correction. From the results, it can be seen that a considerable reduction in time is achieved with percentage as high as $\mathbf{3 7 . 9 4 \%}$. In Table 5, time taken for motion cor- 
Table 3: Entropy values for different motion categories for image resolution at $32 \times 32$ and block size at $8 \times 8$.

\begin{tabular}{|c|c|c|c|c|}
\hline $\begin{array}{c}\text { Motion } \\
\text { Category }\end{array}$ & $\begin{array}{c}\text { Angle of } \\
\text { rotation } \\
\text { in degrees })\end{array}$ & $\begin{array}{c}\text { Peak } \\
\text { Entropy } \\
\left(\mathbf{H}_{\mathbf{p e a k}}\right)\end{array}$ & $\begin{array}{c}\text { Total } \\
\text { Entropy - Unt } \\
\left(\sum_{\mathbf{H}_{\mathbf{n}}}\right)\end{array}$ & $\begin{array}{c}\text { Total } \\
\text { Entropy - V } \\
\left(\sum_{\mathbf{V}_{\mathbf{n}}}\right)\end{array}$ \\
\hline none & 0 & 0.00 & 0.00 & 0.00 \\
\hline minimal & 1 & 0.00 & 0.00 & 0.00 \\
\hline minimal & 2 & 0.00 & 0.00 & 0.00 \\
\hline minimal & 3 & 0.04 & 0.00 & 0.04 \\
\hline minimal & 4 & 0.08 & 0.00 & 0.23 \\
\hline minimal & 5 & 0.20 & 0.00 & 0.76 \\
\hline mild & 6 & 0.25 & 0.00 & 1.29 \\
\hline mild & 7 & 0.40 & 0.00 & 2.04 \\
\hline mild & 8 & 0.52 & 0.00 & 2.67 \\
\hline mild & 9 & 0.61 & 0.00 & 3.25 \\
\hline mild & 10 & 0.75 & 0.08 & 3.78 \\
\hline severe & 11 & 1.05 & 0.32 & 4.33 \\
\hline severe & 12 & 1.15 & 0.48 & 5.14 \\
\hline severe & 13 & 1.31 & 0.59 & 5.75 \\
\hline severe & 14 & 1.37 & 0.85 & 6.21 \\
\hline severe & 15 & 1.51 & 0.97 & 6.88 \\
\hline
\end{tabular}

Table 4: Time analysis for motion detection at different slice resolutions and block sizes. Mean time per slice pair is the sum of time taken for downsampling and deriving $U_{n}$ and $V_{n}$ flow maps.

\begin{tabular}{|c|c|c|c|}
$\begin{array}{c}\text { Slice } \\
\text { Resolution }\end{array}$ & $\begin{array}{c}\text { Block } \\
\text { Size }\end{array}$ & $\begin{array}{c}\text { Mean Time } \\
\text { per Slice Pair(sec) }\end{array}$ & $\begin{array}{c}\text { Total } \\
\text { Time(sec) }\end{array}$ \\
\hline $128 \times 128$ & $32 \times 32$ & $0.00+3.48=3.48$ & 132.21 \\
\hline $128 \times 128$ & $16 \times 16$ & $0.00+3.99=3.99$ & 151.69 \\
\hline $128 \times 128$ & $8 \times 8$ & $0.00+4.34=4.34$ & 164.84 \\
\hline $64 \times 64$ & $16 \times 16$ & $0.01+0.77=0.78$ & 29.71 \\
\hline $64 \times 64$ & $8 \times 8$ & $0.01+0.97=0.98$ & 37.38 \\
\hline $32 \times 32$ & $8 \times 8$ & $0.01+0.19=0.20$ & $\mathbf{7 . 6 8}$ \\
\hline
\end{tabular}

rection with motion detection $\left(t_{n e w}\right)$ is reported for motion correction stage only since the time taken for motion detection $(7.68 \mathrm{sec})$ is negligible compared to the time required for registration.

\section{DISCUSSION AND CONCLUSIONS}

We have presented a method to detect motion between consecutive volumes using block based phase correlation in DSC-MRI perfusion data. The obtained flow maps describe the motion between slices. The net entropy in the flow maps was shown to help distinguish between varying degree of motion corrupted data. The main goal of this approach is to reduce the time for the motion correction process by removing the stationary volumes from the set of volumes to be registered and speed up the final (corrected) data acquisition process. The detection method can be made to be fast however the sensitivity to minimal motions may be compromised.

The emphasis of the work is on an effective motion detection technique which is immune to changes in signal intensity due to injected contrast. The utility of this work in retrospective motion analysis is to improve the time required for motion correction schemes with dynamic perfusion imaging data which currently accounts for as high as $90 \%$ of the data acquisition time. The obtained figures for time savings indi-

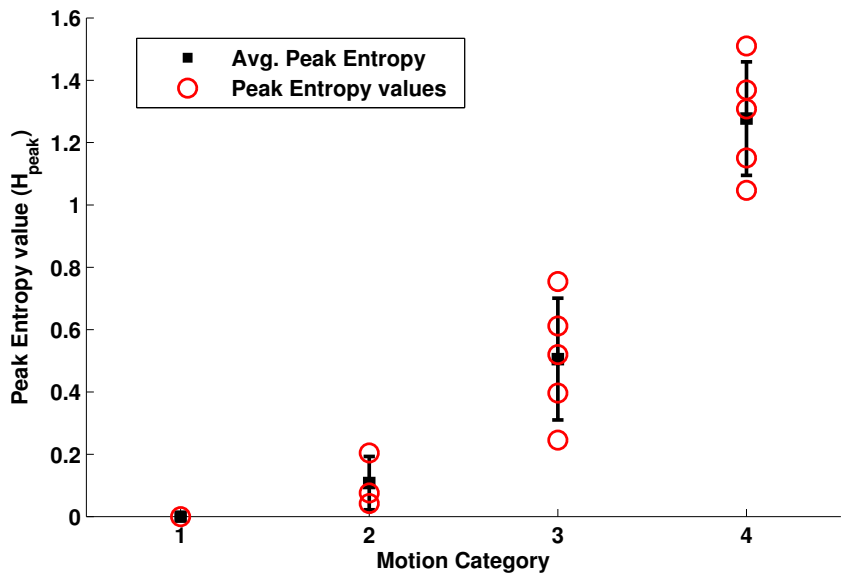

Figure 8: Peak Entropy metric for different motion categories on $\mathrm{N}=16$ datasets. (The values on $\mathrm{X}$ axis ranging from 1 to 4 refer to no motion, minimal motion, medium motion and severe motion).

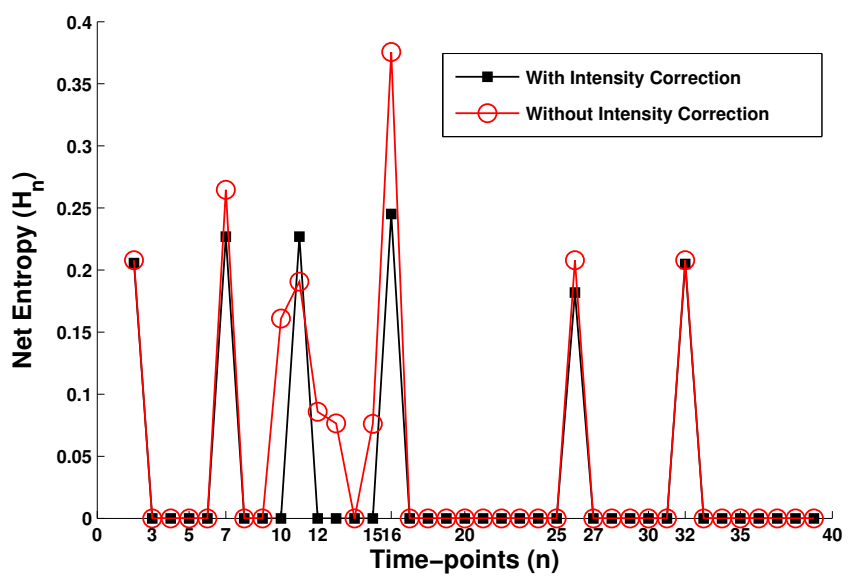

Figure 9: Net entropy $\left(H_{n}\right)$ profile for motion detection with and without using intensity correction.

cates that overall it can be brought down to $53 \%$ even with a MATLAB implementation. A hierarchical implementation with varying slice resolution and the block size can further speed up the process. Another key advantage of employing a motion detection stage in the data acquisition pipeline is that feedback can be provided to the scanner console operator on presence as well as the degree of motion so that appropriate action can be taken.

\section{ACKNOWLEDGEMENTS}

We acknowledge the collaboration between GE Global Research, Bangalore, India and IIIT-Hyderabad, India which facilitated development of the work done in this study. We also thank KIMS hospital, Hyderabad, India for making the perfusion (DSC) MR data available.

\section{REFERENCES}

[1] J. Bican and J. Flusser. 3d rigid registration by cylindrical phase correlation method. Pattern Recogn. Lett., 30:914-921, July 2009. 
Table 5: Evaluation of our method with existing motion algorithms for a DSC-MRI series of $\mathrm{N}=40$ timepoints. Motion was added in $(\mathrm{m}=3)$ intervals. Total no. of volumes to which motion was added: $\mathrm{N}_{1}=16$. After applying motion detection, only $\mathbf{N}_{\mathbf{m d}}=(\mathbf{N}-\mathbf{1})-\mathbf{N}_{\mathbf{1}}+\mathbf{m}$ volumes in the time-series need to be aligned.

\begin{tabular}{|c|c|c|c|c|c|}
\hline $\begin{array}{c}\text { Motion } \\
\text { Merrection } \\
\text { Method }\end{array}$ & $\begin{array}{c}\text { Time taken } \\
\text { to correct (N-1) } \\
\text { volumes - } \mathbf{t}_{\text {old }} \\
(\mathbf{s e c})\end{array}$ & $\begin{array}{c}\text { Mean Time } \\
\text { per volume regn. } \\
\mathbf{t}_{\mathbf{m}}=\mathbf{t}_{\text {old }} /(\mathbf{N}-\mathbf{1}) \\
(\mathbf{s e c})\end{array}$ & $\begin{array}{c}\text { Time taken } \\
\text { to correct } \mathbf{N}_{\text {md }} \\
\text { volumes }-\mathbf{t}_{\text {new }} \\
(\mathbf{s e c})\end{array}$ & $\begin{array}{c}\text { Reduction } \\
\text { in time } \\
\mathbf{t}_{\text {old }}-\mathbf{t}_{\text {new }} \\
(\mathbf{s e c})\end{array}$ & $\begin{array}{c}\text { Percentage } \\
\text { Time Reduction } \\
(\%)\end{array}$ \\
\hline$[10]$ & 640.39 & 16.42 & 397.42 & $\mathbf{2 4 2 . 9 7}$ & 37.94 \\
\hline$[16]$ & 636.38 & 16.32 & 395.74 & $\mathbf{2 4 0 . 6 4}$ & 37.81 \\
\hline$[18]$ & 1018.20 & 26.11 & 668.78 & $\mathbf{3 4 9 . 4 2}$ & 34.32 \\
\hline
\end{tabular}

[2] G. Buonaccorsi, C. Roberts, S. Cheung, Y. Watson, K. Davies, A. Jackson, G. Jayson, and G. Parker. Tracer kinetic model-driven registration for dynamic contrast-enhanced mri time-series data. Magnetic Resonance in Medicine, 58(5):1010-1019, 2007.

[3] A. A. Chan and S. Nelson. Simplified gamma-variate fitting of perfusion curves. International Symposium on Biomedical Imaging, 2(2):1067-1070, 2004.

[4] R. E. and Latchaw. Cerebral perfusion imaging in acute stroke. Journal of Vascular and Interventional Radiology, 15(1, Part 2):S29 - S46, 2004.

[5] A. El-Baz, G. Gimel'farb, and M. El-Ghar. New motion correction models for automatic identification of renal transplant rejection. In Proceedings of the 10th international conference on Medical image computing and computer-assisted intervention, MICCAI'07, pages 235-243, Berlin, Heidelberg, 2007. Springer-Verlag.

[6] C. Grandin. Assessment of brain perfusion with mri: methodology and application to acute stroke. Neuroradiology, 45:755-766, 2003.

[7] M. Jenkinson, P. Bannister, M. Brady, and S. Smith. Improved optimization for the robust and accurate linear registration and motion correction of brain images. NeuroImage, 17(2):825-841, 2002.

[8] M. Kim, G. Wu, and D. Shen. Groupwise registration of breast dce-mr images for accurate tumor measurement. In IEEE International Symposium on Biomedical Imaging, pages 598 -601, 2011.

[9] K. Kohsuke. Perfusion mismatch analyzer. http://asist.umin.jp/index-e.htm, 2011.

[10] R. Kosior, J. Kosior, and R. Frayne. Improved dynamic susceptibility contrast (dsc)-mr perfusion estimates by motion correction. Journal of Magnetic Resonance Imaging, 26(4):1167-1172, 2007.

[11] A. Lausch, M. Ebrahimi, and A. L. Martel. Image registration for abdominal dynamic contrast-enhanced magnetic resonance images. In International Symposium on Biomedical Imaging, pages 561-565. IEEE, 2011.

[12] A. Melbourne, D. Atkinson, M. White, D. Collins, M. Leach, and D. Hawkes. Registration of dynamic contrast-enhanced MRI using a progressive principal component registration (PPCR). Physics in Medicine and Biology, 52(17):5147-5156, Sept. 2007.

[13] L. Ostergaard. Cerebral perfusion imaging by bolus tracking. Topics in magnetic resonance imaging TMRI, 15(1):3-9, 2004 .

[14] G. Rohde, A. Barnett, P. Basser, S. Marenco, and C. Pierpaoli. Comprehensive approach for correction of motion and distortion in diffusion-weighted mri. Magnetic Resonance in Medicine, 51(1):103-114, 2004.

[15] D. Shanbhag, R. Mullick, S. Nath, C. Oppenheim, M. Luby, K. Ku, L. Latour, S. Warach, and N. Natural. Impact of motion and symmetry correction on perfusion lesion segmentation in acute ischemic stroke: Quantitative evaluation. Brain, 17:4710-4710, 2009.

[16] M. Straka, G. Albers, and R. Bammer. Real-time diffusion-perfusion mismatch analysis in acute stroke. Journal of Magnetic Resonance Imaging, 32(5):1024-1037, 2010.

[17] J. L. Sunshine, N. Bambakidis, R. W. Tarr, C. F. Lanzieri, O. O. Zaidat, J. I. Suarez, D. M. Landis, and W. R. Selman. Benefits of perfusion mr imaging relative to diffusion $\mathrm{mr}$ imaging in the diagnosis and treatment of hyperacute stroke. Ajnr American Journal Of Neuroradiology, 22(5):915-921, 2001.

[18] C. Tanner, J. Schnabel, D. Chung, M. Clarkson, D. Rueckert, D. Hill, and D. Hawkes. Volume and shape preservation of enhancing lesions when applying non-rigid registration to a time series of contrast enhancing $\mathrm{mr}$ breast images. In Medical Image Computing and Computer-Assisted Intervention, MICCAI 2000.

[19] S. Thiruvenkadam, S. Arcot, and C. Yunmei. A pde based method for fuzzy classification of medical images. In IEEE International Conference on Image Processing, 2006, pages $1805-1808$, oct. 2006.

[20] J. Tokuda, H. Mamata, R. Gill, N. Hata, R. Kikinis, R. Padera, R. Lenkinski, D. Sugarbaker, and H. Hatabu. Impact of nonrigid motion correction technique on pixel-wise pharmacokinetic analysis of free-breathing pulmonary dynamic contrast-enhanced $\mathrm{mr}$ imaging. Journal of Magnetic Resonance Imaging, 33(4):968-973, 2011. 